Apidologie, 1987, 18 (1), 101-110

\title{
EINFLUSS DER PARASITIERUNG DURCH DIE MILBE VARROA JACOBSONI OUD. AUF DAS SCHLUPFGEWICHT, DIE GEWICHTSENTWICKLUNG, DIE ENTWICKLUNG DER HYPOPHARYNXDRÜSEN UND DIE LEBENSDAUER VON APIS MELLIFERA L. *
}

\author{
Petra SCHNEIDER und Withelm DRESCHER \\ Institut für Landwirtschaftiche Zoologie und Bienenkunde, Melbweg 42, 5300 Bom I
}

\section{ZUSAMMENFASSUNG}

Es konnte gezeigt werden, daß schon durch die Parasitierung weniger Milben meßbare Schäden an den Bienen verursacht werden. Das Schlupfgewicht der Arbeiterinnen wurde durch die Parasitierung eines Mibenweibchens und seiner Nachkommen um $11 \mathrm{mg}(9.6 \%)$ reduzicrt. Befanden sich beim Schlupf der Biene mehr als 3 ausgefärbte Milbenweibchen in der Zelle, war das Gewicht um $24.7 \mathrm{mg}$ $(21.6 \%)$ vermindert. Bei Drohnen betrug diesc Verringerung $9 \mathrm{mg}(3.1 \%)$ bzw. $41 \mathrm{mg}(14.1 \%)$.

Den Gewichtsverlust konnten dic Arbeiterinnen nicht durch dic Haltung in einem varroafreien Bienenvolk kompensieren. Die Gewichtszunahme lag sogar unter der unparasitierter Bienen. Ein Befall durch Varroa-Milben bewirkt auch cine geringere Hypopharynxdrüsenentwicklung. Die Durchmesser der Acini waren nach Befall der Puppen um 13.5\% bzw. $31.5 \%$, durch Schädigung während der Imaginalzeit um $14,6 \%$ verringert. Eine verkürzte Lebensdauer abhängig vom Befallsgrad konnte ebenfalls nachgewiesen werden.

\section{EINLEITUNG}

Die ektoparasitische Milbe Varroa jacobsoni Oud. ernährt sich von der Hämolymphe der Bienenpuppen und der Imagines. Bei starkem Befall während der Puppenentwicklung zeigen die schlüpfenden Bienen Mißbildungen an Abdomen und Flügeln (Haragsim, 1973; DE Jong, MOrSE und Eickwort, 1982).

Aber auch schon ein geringer Milbenbefall bewirkt Schäden. Anshakova et al. (1978) wiesen für Bienen aus Völkern in der UdSSR einen Gewichtsver-

* Gefördert durch das Ministerium für Umwelt, Raumordnung und Landwirtschaft des Landes NRW. 
lust durch Varroa-Parasitierung schon 1977 nach. DE Jong stellte gleiches in Brasilien fest. Er fand bei den dort vorkommenden afrikanisierten Bienen ein niedrigeres Schlupfgewicht und eine verkürzte Lebensdauer nach Varroa-Parasitierung in der Puppenphase (DE Jong et al., 1982; DE JONG und DE JONG, 1983).

Welchen Einfluß eine Parasitierung weniger Varroa-Milben während der Puppenentwicklung auf Apis mellifera im mitteleuropäischen Klimabereich hat, untersuchte bisher nur Weinberg (1985). Er fand eine verminderte Proteinkonzentration in der Hämolymphe von Bienenpuppen in unterschiedlichen Stadien.

Für eine Beurteilung der Schadensschwelle ist es jedoch wichtig, die Beziehung zwischen Milbenbefall und Schaden an der Biene möglichst genau zu kennen. Bei den Untersuchungen sollten klimatische Verhältnisse und Trachtsituation berücksichtigt werden. Die vorliegende Arbeit befaßt sich mit dem Einfluß der Parasitierung auf das Schlupfgewicht, das Gewicht nach 7 Tagen, die Hypopharynxdrüsenausbildung und die Lebensdauer von Bienen (Apis mellifera L.) unter den klimatischen Bedingungen Mitteleuropas.

\section{MATERIAL UND METHODEN}

Als Ausgangsmaterial für dic Versuche dienten Bienen, von denen bekannt war, wieviel Milben auf ihnen während der Puppenentwicklung parasitiert hatten. Das infizierte Versuchsvolk befand sich in einem 10 Waben-Magazin (Normal-Maß) auf zwei Zargen. Aus diesem fand von Mai bis September eine Entnahme von Brutwaben statt, auf denen die Bienen gerade begannen. dic Zelldeckel aufzunagen. Nachdem die Zelldeckel vorsichtig mit einer Pinzette geöffnet waren, zog man dic Bienen heraus und ermittelte die Zahi der ausgefärbten Varroa-Weibchen und Nymphen. Nur Bienen mit sorgfältig kontrolliertem Schlupf wurden berücksichtigt. Die Bienen wurden in 3 Befallsgruppen eingeteilt :

P0 keine Varroa-Milbe während der Puppenphase ;

P1 1 Muttertier mit oder ohne Nachkommen, d.h. die Zahl der ausgefärbten Weibchen betrug 1 bis 3 ; P2 die Zahl der ausgefärbten Weibchen war größer als 3.

Von den geschlüpften Bienen wurden alie Milben entfernt.

\subsection{Gewichtsentwicklung}

Nach Markieren der jungen Bienen wurde das Schlupfgewicht ermittelt und die Bienen in ein varroafreies Völkchen in einem Kirchhainer Kästchen gesetzt. Nach 7 Tagen fand eine erneute Wägung statt. Um die Varroafreiheit des Volkes zu überprüfen, folgte eine Kontrolle auf Milbenbefall bei den entnommenen Bienen.

\subsection{Entwicklung der Hypopharynxdrüsen}

Die Bienen des Wägeversuchs dienten auch zur Bestimmung der Hypopharynxdrüsenentwicklung. Nach dem Abtrennen des Kopfes wurde dieser in Kolophoniumwachs gebettet und sofort tiefgekühlt. Es folgte das Öffnen der Kopfkapsel unter einem Binokular bei 12-facher Vergrößerung und dic Präparation des rechten Drüsenstranges. Dieser wurde in Glyceringelatine gebettet und anschließend die Durchmesser von 40 Acini je Präparat in ihrer Ausprägung parallel zum Drüsenstrang mit Hilfe cines Zeichentubus bei 315-facher Vergrößerung bestimmt. 
Zur Ermittlung des Einflusses einer Parasitierung während der Imaginalzeit erfolgte eine Aufteilung der Bienen der Gruppe P0 und P1 auf 2 Holzkästchen. Die Bienen, die durch Varroa-Milben parasitiert werden sollten, wurden nach dem Schlupf mit Milben infiziert. Die Zahl der zugesetzten Milben war doppelt so groß wie die der Bienen.

Den Bienen stand Futterteig, Wasser und Pollen in ausreichendem Maß zur Verfügung. Die Haltung erfolgte bei $35^{\circ} \mathrm{C}$ und $70 \%$ rel. Lufteuchte im Brutschrank. Nach 3 Tagen erhiclten sie ein Stück Brutwabe in die Holzkästchen auf dem sich Eier und junge Larven befanden. Die Bienen pflegten die jungen Larven weiter. Im Alter von 7 Tage wurden die Beinen nac oben genanter Methode präpariert.

Im folgenden werden die Ergebnisse mit « 10 »ür Bienen, die während der Imaginalphase nicht parasitiert waren und mit «Il » für parasitierte Bienen bezeichnet.

\subsection{Ermittlung der Lebensdauer}

Nach dem Schlupf der Bienen und der Eintcilung in die drei Gruppen folgte eine individuclle Markierung mit nummerierten Opalitplättchen. Die Bienen wurden auf eine Honigwabe mit Bienen aus dem Versuchsvolk gesetzt und diese in einer Wabentasche bei $35^{\circ} \mathrm{C}$ und $70 \%$ rel. Luftfeuchte 24 Stunden in einem Klimaschrank aufbewahrt. Anschließend konnte die Wabe mit aufsitzenden Bienen wieder in das isoliert stehende Volk zurückgegeben werden. Durch diesen eintägigen Aufenthalt im Brutschrank wurden die Anfangsverluste an jungen Bienen erheblich gesenkt.

Dic Zahl der überlebenden markierten Bienen im Versuchsvolk wurde in regelmäßigen Abständen kontrolliert.

\section{ERGEBNISSE}

\subsection{Gewichtsentwicklung}

Das Schlupfgewicht der Arbeiterinnen ist in Tabelle 1 dargestellt. Es betrug bei unparasitierten Bienen durchschnittlich $114 \mathrm{mg}$, bei Bienen der P1Gruppe $103 \mathrm{mg}$, die P2-Gruppe wog $89 \mathrm{mg}$. Die Differenzen sind signifikant $(\mathrm{P}=0,001)$.

Nach 7 Tagen lag das Gewicht der unparasitierten Bienen bei $139 \mathrm{mg}$, Bienen der Gruppe P1 wogen $124 \mathrm{mg}$ und die der Gruppe P2 durchschnittlich $104 \mathrm{mg}$. Auch hier waren die Differenzen signifikant $(P=0,001)$. Die Bienen der Gruppe P1 waren also nach 7 Tagen im Vergleich zu unparasitierten Bienen $11 \%(15,4 \mathrm{mg})$ und die der P2-Gruppe 25,1\% (35,0 mg) leichter.

Auch bei Drohnen wird das Schlupfgewicht durch die Parasitierung vermindert. Die Ergebnisse finden sich ebenfalls in Tabelle 1. Die Unterschiede waren signifikant.

Die Befallsgruppe P1 hat bei Drohnen und Arbeiterinnen eine gleichhohe Milbenzahl. Ein Vergleich der absoluten Gewichtseinbußen bei dieser Gruppe zeigt, daß sie bei Drohnen und Arbeiterinnen annähernd gleich hoch sind. 
TAB. 1. -- Einfluß der Varroa-Parasitierung während der Puppenentwicklung auf das Schlupfgewicht und das Gewicht nach 7 Tagen Aufenthalt im Volk

TABl.. 1. - Influence of Varroainfestation during pupal development on the weight of emerging worker bees and drones and on their weight change

\begin{tabular}{|c|c|c|c|c|c|}
\hline $\begin{array}{l}\text { Parasitierungs- } \\
\text { grad } \\
\text { Infestation rate }\end{array}$ & $\begin{array}{l}\text { Schlupfgewicht } \\
\text { Emergence weight } \\
\text { (mg) }\end{array}$ & $\begin{array}{c}\text { Differenz } \\
\text { zu P0 } \\
\text { Difference from P0 } \\
(\%)\end{array}$ & $\begin{array}{c}\text { Gewicht } \\
\text { nach } 7 \text { Tagen } \\
\text { Weight after } 7 \text { days } \\
\text { (mg) }\end{array}$ & $\begin{array}{l}\text { Differenz zum } \\
\text { Difference from } \\
\text { (mg) }\end{array}$ & $\begin{array}{c}\text { Schlupfgewicht } \\
\text { emergence weight } \\
(\%)\end{array}$ \\
\hline \multicolumn{6}{|c|}{ Arbeiterinnen (worker bees) } \\
\hline $\mathrm{P} 0$ & 114 & & 139 & 25 & 18,0 \\
\hline P1 & 103 & 9,6 & 124 & 21 & 16,9 \\
\hline P2 & 89 & 21.6 & 104 & 15 & 14,4 \\
\hline
\end{tabular}

\begin{tabular}{|c|c|c|c|}
\hline \multicolumn{4}{|c|}{ Drohnen (drones) } \\
\hline PO & 290 & & \\
\hline P1 & 281 & 3,1 & \\
\hline P2 & 249 & 14,1 & \\
\hline \multicolumn{4}{|c|}{$\begin{array}{l}\text { P0 = Unparasitiert während der Puppenphase } \\
\text { No mites during pupal development }\end{array}$} \\
\hline \multicolumn{4}{|c|}{$\begin{array}{l}\text { P1 = 1-3 crwachsene Milbenweibchen in der Brutzelle beim Schlupf der Biene } \\
\text { 1-3 adult female mites in brood cell when bee emerged }\end{array}$} \\
\hline \multicolumn{4}{|c|}{$\begin{array}{l}\text { P2 = Mehr als } 3 \text { erwachsenc Milbenweibchen in der Brutzelle beim Schlupf der Bienc } \\
\text { More than } 3 \text { adult femalc mites in brood cell when bee emerged }\end{array}$} \\
\hline
\end{tabular}

\subsection{Entwicklung der Hypopharynxdrüsen}

Die Hypopharynxdrüsen zeigten abhängig vom Parasitierungsgrad in der Puppenphase eine unterschiedliche Ausbildung (Abb. 1). Die Acini hatten bei der Gruppe P0 eine durchschnittliche Ausbildung parallel zum Drüsenstrang von $114,0 \mu \mathrm{m}$ und bei $\mathrm{P} 1$ von $98,6 \mu \mathrm{m}$. Der Unterschied ist signifikant $(P=0,05)$. Bienen der Gruppe $P 2$ wiesen einen Acinusdurchmesser von $78,1 \mu \mathrm{m}$ auf. Auch dieser Unterschied war statistisch abzusichern $(P=0,001)$. Die Ausbildung der Acini war bei der Gruppe P1 im Vergleich zu unparasitierten Bienen 13,5\% $(15,4 \mu \mathrm{m})$ und bei $\mathrm{P} 2$ um $31,5 \%(35,9 \mu \mathrm{m})$ geringer.

Durch Parasitierung während der Imaginalzeit verringerte sich der Durchmesser der Acini bei Arbeiterinnen der Gruppe P0 von $103 \mu \mathrm{m}$ auf $87,9 \mu \mathrm{m}$ $(\mathrm{P}=0,001)$ und bei $\mathrm{P} 1$ von $95,5 \mu \mathrm{m}$ auf $90,5 \mu \mathrm{m}$ (n.s.). Das heißt, daß durch eine Parasitierung während der Imaginalzeit der Acinusdurchmesser bei der Gruppe P0I1 gegenüber P0I0 um $14,6 \%(15 \mu \mathrm{m})$ und um $5,7 \%(5,4 \mu \mathrm{m})$ zwischen P1I0 und P1I1 verringert wurde. 


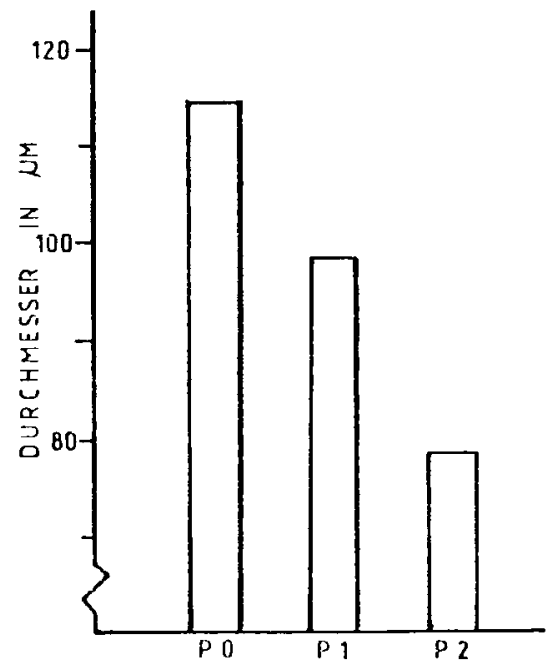

Aвв. 1. - Einfluß unterschiedlicher Varroa-Parasiterung während der Puppenphase auf die Ausprägung der Hypopharynxdrüse (Erklärung des Parasiticrungsgrades s. Tab. 1)

Fig. 1. - Influence of Varroainfestation during the pupal stage on development of hypopharyngeal glands (for explanation sec tabl. 1)

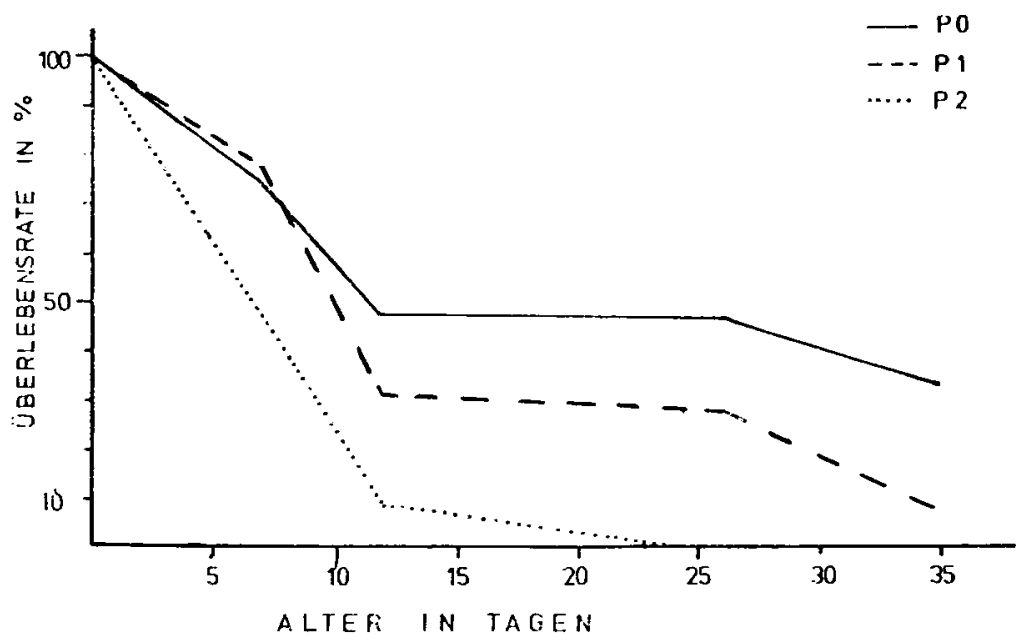

Aвв. 2. - Einfluß unterschiedlicher Varroa-Parasitierung während der Puppenphase auf die Lebensdauer der Arbeiterinnen (Erläutcrung des Parasiticrungsgrades s. Tab. 1)

Fig. 2. - Influence of Varroainfestation during the pupal stage on the longevity of worker bees (for explanation sec tabl. 1) 


\subsection{Einflu $\beta$ der Parasitierung auf die Lebensdauer der Arbeiterinnen}

Wie Abbildung 2 zeigt, verringert die Parasitierung während der Puppenphase die Lebensdauer der Arbeiterinnen. Von den ins Volk gesetzten Bienen (100\%) waren nach 35 Tagen bei der Gruppe P0 noch $33 \%$ zu finden, die Gruppe P1 wies nur noch $8 \%$ der zugesetzten Individuen auf. Aus der Gruppe P2 waren schon 26 Tage nach dem Zusetzen keine Arbeiterinnen mehr zu finden.

\section{DISKUSSION}

Lange bevor es zu sichtbaren Schäden an Bienen (verkürztes Abdomen, verkrüppelte Flügel) und Bienenvolk durch eine hohe Parasitenzahl kommt, sind an den Individuen schon Leistungseinbußen festzustellen.

Die Gewichtseinbußen, die DE JONG et al. (1982) an afrikanisierten Bienen in Brasilien feststellte, sind vergleichbar mit den oben genannten Ergebnissen. Unparasitierte Arbeiterinnen wiesen ein durchschnittliches Schlupfgewicht von $89 \mathrm{mg}$ auf. Bienen, die während der Puppenentwicklung von 1-9 Milben (adulte Weibchen und Nymphen) parasitiert wurden, zeigten Gewichtsverluste von $6,3-25 \%(6,16-23 \mathrm{mg})$.

Bei den hier untersuchten Bienen der Carnica-Rasse, die in ihrem Normalgewicht höher liegen als die afrikanisierten Bienen, ist der Gewichtsverlust mit den von DE JoNG et al. (1982) gefundenen Werten vergleichbar.

Das Schlupfgewicht der Drohnen wird bei gleicher Milbenzahl um annähernd den gleichen absoluten Wert verringert. Eine Erklärungsmöglichkeit wäre, daß die Milbenweibchen von Arbeiterinnen- und Drohnenpuppen die gleiche Hämolymphmenge aufnehmen, die Qualität der Nahrung für die Milben also vermutlich gleich ist.

Der durch die Parasitierung hervorgerufene Schaden kann von den Bienen durch ein varroafreies Leben nach dem Schlupf im Volk nicht kompensiert werden. Ob die Schädigung ausschließlich durch Hämolymphentzug verursacht wird oder noch andere Komponenten, wie Abgabe toxischer Substanzen oder Beunruhigung der Larve, hinzukommen, muß noch geklärt werden. Diese Schädigung bewirkt sogar eine verringerte Gewichtszunahme, eine verringerte Hypopharynxdrüsenentwicklung und eine verkürzte Lebensdauer während der Imaginalphase. Letztere wird unter unseren klimatischen Verhältnissen vor allem bei der Überwinterung zum Problem. Haben die Bienen eine verkürzte Lebensdauer, wie dies auch bei Parasitierung durch die Tracheenmilbe der Fall ist, kommt es im Frühjahr zu Volkszusammenbrüchen (BAILEY, 1981). 
Die Verkürzung der Lebensdauer ist ebenfalls vergleichbar mit den Ergebnissen, die DE JoNG et al. (1983) in Brasilien fanden. Lebensdauer und physiologischer Zustand (Entwicklung der Hypopharynxdrüsen, Fettkörperausbildung) sind entscheidend abhängig von der Eiweißversorgung der Biene (Maurizio, 1954 ; Knox et al., 1971). Eine Parasitierung der Bienenpuppe durch 1-3 Milben verringert die Proteinkonzentration in der Hämolymphe bei schlupfbereiten Bienen um 6,24 \%, durch 4-6 Milben um 11,19\% (WEINBERG, 1985). Es wäre möglich, daß dieser Proteinentzug die Ursache für die verkürzte Lebensdauer und die geringere Hypopharynxdrüsenentwicklung ist. Ein zusätzlicher Proteinentzug im Imaginalstadium durch Parasitierung bzw. ein Pollenmangel im Volk dürften diese Wirkung noch verstärken.

Es bleibt die Frage, wie sich die Parasitierung auf die Pflegeleistung und die Qualität des Futtersaftes auswirkt. Brouwers (1982) fand heraus, daß zwischen Größe der Acini und Futtersaftproduktion kein Zusammenhang besteht.

Eingegangen im März 1986. Angenommen im Mai 1986.

\section{SUMMARY \\ THE INFLUENCE OF VARROA JACOBSONI OUD. ON WEIGHT, DEVELOPMENT OF WEIGHT AND HYPOPHARYNGEAL GLANDS, AND LONGEVITY OF APIS MELLIFERA L.}

The damage to worker bees and drones from infestation with the parasitic mite Varroa jacobsoni Oud. in central Europe was investigated. Not only do high infestation rates of mites cause damage to the becs ; 1-3 mites/pupac result in detectable negative effects.

This study was conducted with bees where the exact number of parasitizing mites during pupal development was determined. Brood cells from combs which were kept in an incubator were opened before emergence and the bees collected. The mites on the emerging bee and those remaining in the brood cell were counted. The newly emerged bees were marked, weighed and kept in a colony without Varroamites.

After 7 days the bees were weighed again. Also the complex of their hypopharyngeal gland was prepared and the diameter of 40 acini was measured.

To compare longevity newly emerged bees with different degrees of parasitization during pupal development were marked individually and released into an isolated colony without Varroamites. The presence of the bees was recorded regularly.

The mean weights of emerging bees were reduced by $11 \mathrm{mg}(9,6 \%)$ when there was one female mite with her offspring on the bee pupac. The reduction was $24,7 \mathrm{mg}(21,6 \%)$ when there were more than 3 adult mites. Average weight loss of emerging drones infested by one adult mite with her offspring was $9 \mathrm{mg}(3,1 \%)$ when infested by more than 3 it was $41 \mathrm{mg}(14,1 \%)$ (Tabl. 1).

Worker bees are not able to compensate for weight losses caused by Varroainfestation when they develop in a colony without mites. Weight pain is less for parasitized workers than for bees which were not parasitized by mites during pupal stage (Tabl. 1).

Mite infestation reduces the size of the hypopharyngeal glands too. When bees were damaged during pupal stage the size of the acini was reduced by $13,5 \%$ and $31,5 \%$ respectively, for infestation of $1-3$ 
more than 3 adult female mites (Fig. 1). Infestation of adult worker bees reduced the size of the glands by an average of $14,6 \%$.

It was also shown that the longevity of adult worker bees was reduced when bees are infested during pupal development (Fig. 2).

It may be that the loss of protein caused by Varroainfestation is the most important factor for the reported damages. Our results dealing with weight loss and longevity are in agrecment with the results reported by DE JoNG et al. $(1982,1983)$ for Brazil.

\section{RÉSUMÉ \\ ACTION DE VARROA JACOBSONI OUD. SUR LE POIDS À L'ÉMERGENCE, LE DÉVELOPPEMENT PONDÉRAL ET DES GLANDES HYPOPHARYNGIENNES ET LA LONGÉVITÉ D'APIS MELLIFICA $L$.}

On a ćtudié les dégâts causés en Europe centrale aux ouvrières et aux mâles par l'acarien parasite Varroa jacobsoni Oud. Des taux élevés d'infestation ne sont pas seuls à causer des préjudices aux abeilles; 1 à 3 acariens/nymphe suffisent à provoquer des effets négatifs décelables.

Cette étude a porté sur des abeilles dont on avait déterminé le nombre exact d'acariens présents au cours du développement nymphal. Les cellules de couvain, provenant de rayons conservés à l'étude, ont été ouvertes avant l'émergence et les abeilles récoltées. On a dénombré les acariens présents sur les abcilles naissantes et ceux restant dans la cellule. Les abeilles fraîchement écloses ont été marquées, pesées et conservées dans une colonie dépourvues de Varroa.

Au bout de 7 jours on a repesé les abeilles. On a également préparé leurs glandes hypopharyngiennes et mesuré le diamètre de $\mathbf{4 0}$ acini. Afin de comparer la longévité, des abeilles fraîchement écloses, présentant différents degrés d'infestation au cours du développement nymphal, ont été marquées individuellement et relâchées dans une colonie isolée dépourvue de Varroa.

Un seul acarien femelle, et sa descendance, a fait baisser le poids moyen des abeilles naissantes de $11 \mathrm{mg}(9,6 \%)$. La diminution de poids a atteint $24,7 \mathrm{mg}(21,6 \%)$ lorsqu'il y avait plus de 3 acariens adultes. La perte moyenne de poids des mâles naissants infestés par un acarien adulte, et sa descendance, a été de $9 \mathrm{mg}(3,1 \%)$ et de $41 \mathrm{mg}(14,1 \%)$ lorsqu'il y en avait plus de 3 (Tabl. 1).

Les abeilles ouvrières n’ont pas été capables de compenser les pertes de poids causées par Varroa lorsqu'elles se sont développées dans une colonie sans acariens. Le gain de poids a été moindre pour les ouvrières parasitćes que pour celle qui n'avaient pas été parasitćes au cours du stade nymphal (Tabl. 1).

L'infestation par les acariens a réduit également la taille des glandes hypopharyngiennes. Lorsque les abeilles ont été endommagées durant le stade nymphal, la taille des acini a été réduite de $13,5 \%$ et de $31,5 \%$ pour des infestations respectives de 1 à 3 et de plus de 3 acariens femelles adultes (Fig. 1). L'infestation d'abeilles ouvrières adultes a réduit la taille des glandes de $14,6 \%$ en moyennc.

On a aussi montré que la longévité des abeilles ouvrières adultes était réduite lorsqu'elles étaient infestées pendant leur développement nymphal (Fig. 2).

Il est possible que la perte de protéines provoquée par l'infestation par Varroa jacobsoni soit le facteur le plus important des dégâts observés. Nos résultats concernant la perte de poids et la longévité sont en accord avec ceux de DE Jong et al. (1982, 1983) pour le Brésil.

\section{LITERATURVERZEICHNIS}

Anshakova O.V., Bobxova V.V., Grobov O.F., Korjova L.M., Langhe A.B., Mikituk V.V., NaTzki K.V., Stolnov N.M., 1978. - Contributions to biological study of Varroa jacobsoni and its influence on honey bees. XXVI Int. Beekeep. Congr., Adelaide, Australia, 439-441. 
Bail.ey L., 1981. - Honey Bee Pathology. Academic Press Inc., London, 601-67.

Brouwers E.V.M. 1982. - Measurement of hypopharyngeal gland activity in the honcybec. J. apic. Res., $21,193-198$.

DE Jong D., DE Jong P.H., 1983. - Longevity of africanized honcy becs (Hymcnoptcra : Apidac) infested by Varroa jacobsoni (Parasitiformes: Varroidae). J. Econ. Entomol., 76. 766-768.

DE Jong D., de Jong P.H.. Gonçal.ves L.S., 1982. - Weight loss and other damage to developing worker honeybees from infestation with Varroa jacobsoni. J. apic. Res., $21,165-167$.

De Jong D., Morsf R.A., Eickwort G.C., 1982. - Mite pests of honeybees. Annu. Rei. Entomol. . 27. 229-252.

Haragsim O., 1973. - Dic Milbe Varroa jacobsoni Oudemans bedroht dic Bienenzucht in Europa. Imkerfreund, $28,316-317$.

Maurizio A.. 1954. - Pollenemährung und Lebensvorgänge bei der Honigbienc (Apis mellifera L.). Landwirtschaftl. Jahrbuch der Schweiz, 3 (68), 115-183.

Knox D.A., Shimanukı H., Herbert E.W., 1971. - Diet and the Longevity of Adult Honey Bees. J. Econ. Entomol., 64. 1415-1416.

Weinberg K.P., Madel. G., 1985. - The Influence of the Mite Varroa jacobsoni Oud. on the Protein Concentration and the Hacmolymph Volume of the Brood of Worker Bees and Drones of the Honcy Bce Apis mellifica L. Apidologie, 16 (4), 421-436. 